\title{
Visual and tactile scanning: Moving scan versus moving medium
}

\author{
NICHOLAS C. NOLL and ROBERT J. WEBER \\ Oklahoma State University, Stillwater, Oklahoma
}

\begin{abstract}
Two models of scanning are examined: a moving attention focus (and fixed spatial medium) versus a moving medium (and fixed focus of attention). These are referred to respectively as moving scan and moving medium models. Kosslyn (1980) has argued for a moving medium model in visual imagery. These models were tested by examining distance estimates of the separation of two points on a map as perceived through an aperture in visual and tactile modes. In the first experiment, viewing a map moving behind a stationary aperture (moving medium condition) led to significantly less accurate spatial distance judgments than did viewing the aperture moving over the map (moving scan condition). In the second experiment, vision and touch were compared. Distance estimates were larger for the moving scan condition than for the moving medium condition. Distance estimates were also larger for visual than for touch conditions. Moreover, modality did not interact with the manipulation of moving medium versus moving scan. It was concluded that (1) reception and representation of information through an aperture involves a general type of processing not specific to any one sensory modality, and (2) strongest support is found for the moving scan model in which an attention pointer moves across a fixed medium.
\end{abstract}

Kosslyn (1980) suggested that scanning to a target object in a visual image does not involve moving an attention pointer across the image but instead is the movement of the image under a "central . . . highly resolved region" (p. 329). The perceptual analogy would be eyes moving over a fixed visual spatial medium versus a moving medium that passes under a fixed eye position. The latter alternative corresponds to watching objects in a rearview mirror or moving data in and out of view on a spread sheet. In a formal sense, the two models are probably equivalent. In fact, the primary justification for Kosslyn's acceptance of the moving medium alternative would seem to be computational simplicity in his simulation of the imagery system. Psychologically, however, the two models seem to pose a fundamental distinction between scanning mechanisms.

A very simple situation is examined to study the alternative conceptions of scanning: distortions of distance between two points. This situation is simple, quantitative, and allows for the testing and direct comparison of scan mechanisms in both the visual and tactile modalities. It is assumed that the natural scan mode is that condition associated with the smallest error in distance judgment. The two points are observed in visual or tactile mode through a narrow aperature that moves over them (moving scan condition), or they may be viewed through a fixed aperture under which the map on which points are placed is moved (moving medium condition). It is assumed that the aperture condition, which is most accurate, reflects

R. J. Weber's mailing address is: Psychology Department, Oklahoma State University, Stillwater, OK 74078. the true scanning mechanism for the conditions studied.

Precedent for studying image representation by using aperture techniques comes from the work on left neglect by Bisiach and his collaborators. Bisiach, Luzzatti, and Perani (1979) examined right-brain-damaged patients suffering left neglect. Cloud-shaped forms were rear projected on a screen. The phenomenal appearance was that of the clouds passing behind a narrow aperture. The left/ right ends of each cloud carried the critical shape information. For a pair of successively presented clouds, the two left ends might be the same or different, and the two right ends might be the same or different. The subject's task was to indicate whether a pair of clouds were overall the same/different. Findings were that differences on the left ends of clouds were poorly detected relative to differences on the right ends of clouds. Poor detection of differences was markedly greater for the right-braindamaged subjects than for a normal control group, but the two groups were similar in detecting differences on the right ends of the clouds. Because all information was presented through a narrow fixed slit, it follows that perceptual input was not involved and that it was the mental representation of leftness information that was impaired in the brain-damaged patients. The nature of this representation would seem at some level to be a visual image. Support for such a conclusion comes from Bisiach and Luzzatti (1978) who had left-neglect patients imagine a public square from two diametrically opposite views. In each case, the patients described primarily buildings or objects on the right and failed (neglected) to mention things on the left. This could not be a general storage or recall problem because the buildings neglected from one imaginal vantage point were correctly described from the op- 
posite view, and vice versa. What these studies together indicate is that mental representation can be built up from aperture exposure and that those representations include visual image components. If that is the case, aperture studies may then be used to determine whether acquiring mental image representations and accessing information from them by scanning varies according to whether the scan moves over a fixed medium or a moving medium passes under a fixed scan.

The use of apertures to study perception has a long history. Zöllner (1862) first studied perception through a narrow opening, using the phrase "anorthoscopic perception" to describe this visual situation. Zöllner passed simple figures behind a stationary aperture. He noted two basic results (1) When a figure is moved behind a stationary aperture at relatively fast speed (less than $1 \mathrm{sec}$ per pass), an extended figure is perceived in the vicinity of the aperture. (The entire figure appears to be visible at one time, and the figure is compressed horizontally.) (2) When a figure is moved behind the aperture at relatively slow speed (greater than $1 \mathrm{sec}$ per pass), the figure is perceived section by section and appears elongated horizontally. Zöllner did not study the situation in which an object moves behind a stationary aperture. Parks (1965) has recently studied a similar phenomenon.

More recently, a number of studies have investigated perception through apertures under fast stimulus-movement conditions (Anstis \& Atkinson, 1967; Haber \& Nathanson, 1968; Rock \& Sigman, 1973). These studies all found that when a figure moves behind a stationary aperture at fast speed, the figure is perceived as being compressed horizontally. These studies for the most part have employed complex figural stimuli. This has made it difficult to precisely quantify distortion of distance. Also, the complexity of stimuli certainly does not allow for comparison of visual and tactile modes.

A few studies have looked at the case in which the aperture moves in front of a stationary figure (Rock, 1981; Rock \& Sigman, 1973); however, we know neither of studies that have examined distance estimates under such a moving scan condition, nor of studies in which a direct comparison is made between the visual and the tactile modes.

The present experiments are designed to accomplish the following objectives: (1) directly compare distance representation under the moving scan and moving medium conditions; (2) measure distortions in judgment that result from viewing objects at slow speed, where the orientation of movement varies with respect to the body, and (3) determine the generality of scan mode effects by studying two different spatial modalities, vision and touch.

\section{EXPERIMENT 1}

In the first experiment, subjects viewed a series of twopoint maps through 2 -cm-wide $\times 10$-cm-high slits in one of two conditions. In the moving scan condition, subjects viewed the aperture moving over a series of stationary maps, and in the moving medium condition, subjects saw the series of maps move behind a stationary aperture. The rate of movement in either condition was $5 \mathrm{~cm} / \mathrm{sec}$.

In addition, an attempt was made to measure the homogeneity of the visual field under scanning by having each subject view the apparatus in three orientations, with the plane of apparatus movement oriented horizontally, diagonally, or vertically from the subject's perspective. If the scan of the visual field is not homogeneous, then one would expect different results for the three orientations. The estimated distance between the two points of a map served as the response measure.

\section{Method}

Subjects. Subjects were 36 undergraduates from psychology classes at Oklahoma State University who received extra credit for participating. Sixteen males and 20 females were involved in the experiment; they ranged in age from 18 to 29.

Apparatus. The apparatus consisted of two platforms, each $30 \times$ $65 \mathrm{~cm}$, one situated above the other, and each capable of independent movement. A drawing of the apparatus is included in Figure 1. The top platform had a $2 \times 10 \mathrm{~cm}$ aperture cut in it, through which the second platform could be observed. The second platform, which was $1 \mathrm{~cm}$ below the first, was constructed so that a $25 \times 10 \mathrm{~cm}$ map could be attached quickly to it. The apparatus also had a screen (not shown) attached to one end, which concealed the maps when they were changed.

Each map consisted of two rubber faucet washers, $1.1 \mathrm{~cm}$ diam, fastened to a sheet of $25 \times 10 \mathrm{~cm} 3-\mathrm{M}$ brand 100 -grade medium sandpaper. This sandpaper provided a textured background for the maps. The distances between the two washer points were determined randomly with the restriction that the distance was no less than $5 \mathrm{~cm}$ and no greater than $25 \mathrm{~cm}$. Ten maps were created in this manner and presented in random order.

The speed of movement of the aperture or of the map beneath it (depending on the condition) was regulated by the experimenter at $5 \mathrm{~cm}$ per sec. The top platform had a small experimenter's window cut in it through which a series of marks on the lower platform could be viewed by the experimenter. The marks were $5 \mathrm{~cm}$ apart, and the experimenter timed the movement of either the aperture (top platform) or the map (bottom platform) so that the appearance of each mark in the window coincided with a sequence of 1-per-sec tones that the experimenter heard through a set of headphones.

Subjects made their estimates as to the distance between the points by adjusting the distance between two washers on a separate response apparatus to match the distance they had perceived. Three separate response configurations-horizontal, vertical, and diagonal-were used to match the three apparatus orientations seen by each subject.

\section{Results}

The principle results are shown in Figure 2. The results are reported as mean error scores, which are derived by subtracting the actual map distances from the subject's estimate of those distances. An ANOVA indicated that scan condition (moving scan vs. moving medium) was sig-

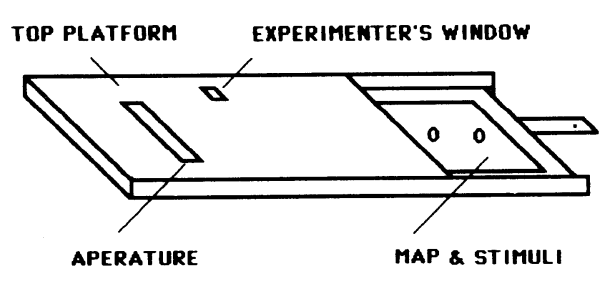

Figure 1. Schematic representation of apparatus. 


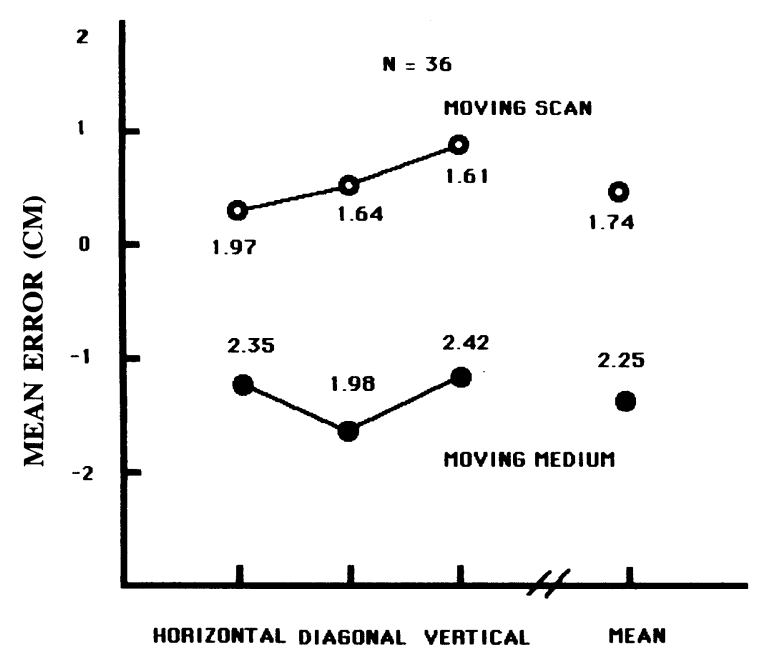

APPARATUS ORIENTATION

Figure 2. Experiment 1: mean error as a function of scan condition and apparatus orientation. SDs beside each point.

nificant $[\mathrm{F}(1,34)=8.051, \mathrm{p}<.05]$. The orientation variable was also significant $[\mathrm{F}(2,68)=3.34, \mathrm{p}<.05]$. The interaction of orientation by scan condition was not significant. The mean error score for the moving medium conditions was $-1.33 \mathrm{~cm}$, indicating that subjects on the average underestimated distances between objects in this condition. A t-test for difference from a population mean of zero indicates that this underestimation is significant $[t(17)=2.964, p<.01]$. The mean error score for the moving scan condition was $0.45 \mathrm{~cm}$, which is not significantly different from zero $[\mathrm{t}(17)=1.204, \mathrm{p}<.05]$.

A series of Newman-Keuls multiple range tests were used to analyze the orientation variable. In the moving scan condition, the vertical apparatus orientation was significantly different $(p<.05)$ from the horizontal orientation (difference $=.869, \mathrm{c}$. diff. $=.816)$ and from the diagonal orientation (difference $=.737$, c. diff. $=.680$ ). In contrast, for the moving medium conditions, no significant differences between apparatus orientation were found.

\section{Discussion}

The moving scan and moving medium conditions were shown to be significantly different from one another. Although scores in the moving scan condition showed a slight overestimation, the mean error score for this condition was not significantly different from zero (zero being the average error score one would expect if no distortions occurred). In contrast, the moving medium condition produced judgments that were significantly less than zero. The results indicate that (1) the distinction between a moving scan and a moving medium is a real difference and not just a matter of formal convenience, and (2) the most strongly supported alternative is the moving scan model. Those investigators simulating the visual imagery system may wish to include specific assumptions in favor of a moving scan process.

The results for the moving medium condition are consistent with what has been called a "compression effect": Distances are perceived as being compressed horizontally, with the average estimate being $81.5 \%$ of the actual map distance. This result constrasts with earlier experimental results reported by Zöllner (1862) and Wenzel (1926). Early research, which looked only at the moving medium situation, made a distinction between fast and slow anorthoscopic perception. A compression effect was reported to occur in fast viewing situations, while an elongation effect sometimes occurred in slow anorthoscopic perception. However, in the present study, slow movement yields a compression effect in the moving scan condition, much the same as is found in fast anorthoscopic perception. One reason for this discrepancy may be the type of responses required from the subjects by the various studies. Both Wenzel (1926) and Zöllner (1862) had observers simply give their impressions of what they saw, but in the present study, subjects were required to make actual estimates of the distances in question. Thus one would expect the present study to give a more accurate measure of error. A second difference between the present study and prior research is the present use of simple stimuli with a textured background (sandpaper). The texture cues may have assisted subjects in making their distance estimates. In contrast, the earlier studies apparently used complex stimuli on untextured backgrounds.

The apparatus orientation variable also yielded some interesting results. In the moving scan condition, maps in the vertical orientation were seen as larger than those in the other two orientations. This effect is similar to the vertical-horizontal illusion, where a vertical line appears to be longer than a physically equal horizontal line. The cause for both the vertical-horizontal illusion and the elongation seen in the vertical orientation of the moving scan condition may be the shape of the visual field. If the visual field is oblong, wider than it is high, then a vertical line or a distance between points would appear to take up more of the visual field than a horizontal line of the same length. For example, a given distance may occupy $50 \%$ of the vertical extent of the visual field, but that same line horizontally oriented may take up only $35 \%$ of the larger horizontal extent of the visual field. If distance perceptions do not take into account the different scale factors (as they do not seem to do because of the horizontal-vertical illusion), then a given distance would appear longer in a vertical than in a horizontal direction. In further support of this view is the finding that, in the moving medium condition, there are no significant differences between apparatus orientations.

\section{EXPERIMENT 2}

The second experiment explores scanning methods in the touch modality. A determination of the generality of scan effects is in order. The question is similar: What scan mode is most natural for the tactile mode, a moving scan or a moving medium?

In the tactile conditions, subjects estimated the distance between the pair of points by the sense of touch, either by moving an index finger across a stationary map (i.e., the moving scan condition) or with the map moving underneath the stationary index finger (i.e., the moving medium condition). Apparatus orientation was not varied, remaining horizontal in all conditions. In the corresponding visual conditions, subjects again estimated the distance between two points on a map, viewed in either the moving scan condition or the moving medium condition.

\section{Method}

Subjects. Subjects were 48 undergraduates from psychology classes who received extra credit for participating. Twelve males and $36 \mathrm{fe}-$ males participated; their ages ranged from 18 to 50 .

Apparatus. The apparatus for presentation of stimuli in visual conditions was the same as described in Experiment 1, with slight modifications for the tactile conditions. The top platform was reinforced so that subjects could rest their hands upon it. A $2 \times 2 \mathrm{~cm}$ opening was cut in the platform, through which the subjects placed the index finger of the dominant hand. Through this opening, subjects felt the maps in the tactile conditions. In addition, a removable screen was constructed to block the subject's view of the apparatus during tactile trials. 
Subjects saw or felt the same 10 map distances used in Experiment 1. In the visual conditions, each map again consisted of two $1-\mathrm{cm}$ rubber washers attached to a $10 \times 25 \mathrm{~cm}$ piece of $3-\mathrm{M}$ brand 100 -grade medium sandpaper. In the tactile conditions, the same distances were used but the points fixed to the sandpaper were two $1-\mathrm{cm}$ flat plastic buttons instead of washers. Buttons were used because it was easier for the subject's index finger to slip across a button than a rubber washer.

The response apparatus was the same as in the first experiment except that only the horizontal orientation was used. Again, the presentation rate of $5 \mathrm{~cm}$ per $\mathrm{sec}$ was held constant across trials.

Procedure. Subjects were randomly assigned to one of four conditions: (1) visual modality/moving medium, (2) visual modality/moving scan, (3) tactile modality/moving medium (stationary finger), or (4) tactile modality/moving scan (moving finger). Subjects were told that they would see/feel a series of maps through the aperture and should adjust the distance between the two points on the response apparatus to match what they saw/felt. Subjects were allowed to inspect an example of the maps for $30 \mathrm{sec}$ in preliminary training.

Subjects in all conditions were exposed to three blocks of 10 maps each. The order of presentation of maps was determined randomly and was the same for all subjects to avoid the difficulty of rearranging the apparatus.

\section{Results}

Due to a great deal of variability in the estimates of the map distances, individual responses greater than two standard deviations above or below the mean for the group were dropped from the analysis. The principal findings are shown in Figure 3. The adjusted mean error scores in centimeters for the four groups were as follows visual/ moving scan $=.59$; visual $/$ moving medium $=-.38$; tactile/moving scan $=-.50$; tactile/moving medium $=$ -1.32. An ANOVA performed on the data indicated that, in both touch and vision, moving medium estimates were significantly less than moving scan estimates $[\mathrm{F}(1,44)=$ $4.38, \mathrm{p}<.05$ ]. Visual estimates were also found to be significantly larger than touch estimates $[\mathrm{F}(1,44)=5.30$, $\mathrm{p}<.05]$. The interaction between mode of presentation and modality used was not significant $[\mathrm{F}(1,44)=.01$,

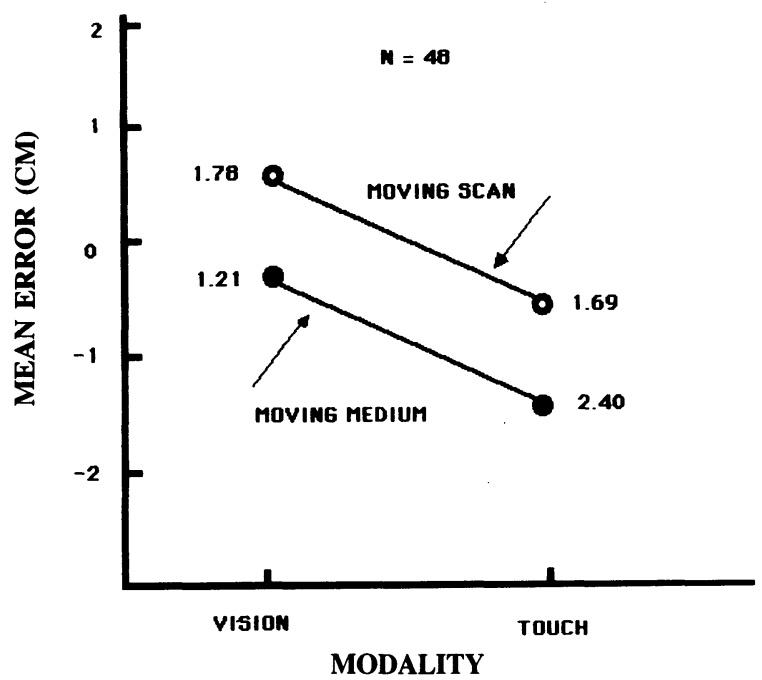

Figure 3. Experiment 2: mean error as a function of scan condition modality. SDs beside each point. $p<.05]$. To examine absolute error, a t-test for the difference from a population mean of zero was performed for each of the four groups, with no significant differences being found.

\section{Discussion}

The results show that a similar pattern of scan effects occurs in both vision and touch. The moving medium conditions produce a compression of judged distances relative to the moving scan conditions. The significant scan effect, along with the lack of an interaction between modality and mode of presentation, indicates that aperture situations are judged in much the same way in vision and in touch. This suggests that the cause of scan effects involves processes that transcend specific modalities.

The fact that touch and vision behave similarly (except for absolute error rates) supports the notion that it is proprioceptive information that is responsible for the improved performance in the moving scan condition. The moving scan conditions in both touch and vision are similar in that they have greater proprioceptive input than the moving medium conditions. In vision, this proprioceptive information would come from eye movements; in touch, the information would come from the movement and positioning of the joints of the wrist and arm.

Although scan effects were similar in pattern for each modality, the absolute effects for vision and touch were not identical. The two modes were significantly different, with the vision effect being closer to the actual distances (zero error) than the touch effect.

A troubling note is the substantial variability encountered. To confuse easy interpretation, the t-tests for differences from a population mean of zero were not significant, attesting to a lack of robustness of the scan effects under the conditions studied. Furthermore, the results for the visual mode are not entirely consistent with the results from Experiment 1 . Thus the results of Experiment 1 suggest a moving scan as the true mode of scanning, because the smallest absolute error is associated with such a scan mode. The results of Experiment 2 suggest that visual and tactile scanning follow the same general pattern, and the absolute errors are all indistinguishable from zero.

\section{REFERENCES}

Anstis, S. M., \& AtKInson, J. (1967). Distortions in moving figures viewed through a stationary slit. American Journal of Psychology, 80, 572-585.

Bisiach, E., \& LuzzatTi, C. (1978). Unilateral neglect of representational space. Cortex, 14, 129-133.

Bisiach, E., Luzzatti, C., \& Perani, D. (1979). Unilateral neglect, representational schema and consciousness. Brain, 102, 609-618.

Haber, R. N., \& NATHANSON, L. S. (1968). Post-retinal storage? Some further observations on Park's camel as seen through the eye of a needle. Perception \& Psychophysics, 3, 349-355.

Hecht, H. (1924). Die simultane Erfassung der Figuren. Zeitschrift für Psychologie, 94, 153-194.

Kosslyn, S. K. (1980). Image and mind. Cambridge, MA: The Harvard University Press.

PARKS, T. E. (1965). Post-retinal visual storage. American Journal of Psychology, 78, 145-147.

Rock, I. (1981). Anorthoscopic perception. Scientific American, 244, 145-153.

Rock, I., \& Sigman, E. (1973). Intelligence factors in the perception of form through a moving slit. Perception, 2, 357-369.

Wenzel, E. L. (1926). Die sukzessive Erfassung de- Figuren. Zeitschrift für Psychologie, 100, 289-324.

ZöLlNER, F. (1862). Über eine neue Art anorthoskopischer Zerrbilder. Annalen der Physik und Chemie, 117, 477-484. 